\title{
BMJ Open Gustatory and olfactory dysfunctions in hospitalised patients with COVID-19 pneumonia: a prospective study
}

\begin{abstract}
Alexy Inciarte (10 , ${ }^{1,2,3}$ Celia Cardozo, ${ }^{1}$ Mariana Chumbita, ${ }^{3}$ Pau Alcubilla, ${ }^{1}$ Berta Torres, ${ }^{1,2}$ Ana González Cordón, ${ }^{1,2}$ Veronica Rico, ${ }^{1}$ Daiana Aguero, ${ }^{1}$ Nicole García-Pouton, ${ }^{1}$ Marta Hernández-Meneses, ${ }^{1}$ Laia Albiach, ${ }^{3}$ Fernanda Meira, ${ }^{3}$ Lorena De la Mora, ${ }^{1,2,3}$ laura Linares, ${ }^{3}$ Pedro Puerta-Alcalde, ${ }^{1}$ Irene Macaya, ${ }^{1}$ Josep Mensa, ${ }^{1}$ Montse Laguno, ${ }^{1,2}$ Juan Ambrosioni, ${ }^{1}$ Angela Ramos, ${ }^{1}$ Laura Morata, ${ }^{1,2}$ Marta Bodro, ${ }^{1,2}$ Estela Moreno-García (i) , ${ }^{1}$ Antonio Moreno, ${ }^{1}$ Montse Sola, ${ }^{1}$ Jhon Rojas, ${ }^{1}$ Lorna Leal, ${ }^{1,3}$ Manuel Torres, ${ }^{1}$ Carolina Garcia-Vidal, ${ }^{1,2}$ Jose Antonio Martínez, ${ }^{1,2}$ Isam Alobid, ${ }^{4}$ Alex Soriano, ${ }^{1,2}$ Felipe Garcia ${ }^{1,2,3}$
\end{abstract}

To cite: Inciarte A, Cardozo C, Chumbita M, et al. Gustatory and olfactory dysfunctions in hospitalised patients with COVID-19 pneumonia: a prospective study. BMJ Open 2021;11:e040775. doi:10.1136/ bmjopen-2020-040775

- Prepublication history for this paper is available online. To view these files, please visit the journal online (http://dx.doi. org/10.1136/bmjopen-2020040775).

Received 21 May 2020 Accepted 28 April 2021

\section{Check for updates}

(C) Author(s) (or their employer(s)) 2021. Re-use permitted under CC BY-NC. No commercial re-use. See rights and permissions. Published by BMJ.

${ }^{1}$ Infectious Diseases Department, Hospital Clinic de Barcelona, Barcelona, Spain ${ }^{2}$ Faculty of Medicine and Health Sciencies, University of Barcelona, Barcelona, Spain ${ }^{3} \mathrm{AIDS}$ and HIV infection, Institut d'Investigacions Biomèdiques August Pi i Sunyer (IDIBAPS), Barcelona, Catalunya, Spain ${ }^{4}$ Rhinology Unit \& Smell Clinic, ENT Department, Hospital Clinic de Barcelona, Barcelona, Spain

Correspondence to

Felipe Garcia;

FGARCIA@clinic.cat

\section{ABSTRACT}

Importance Identifying undetected clinical signs is imperative in the prevention of SARS-CoV-2.

Objective To establish the prevalence of clinical gustatory and olfactory dysfunctions in patients with COVID-19 pneumonia. Clinical outcomes and recovery rates associated with gustatory and olfactory dysfunctions were also assessed.

Design A prospective study was performed in 80 patients admitted to Hospital Clínic of Barcelona (Spain) for COVID-19 pneumonia. Patients were re-evaluated in the ward daily until discharge. Gustatory and olfactory dysfunction symptoms were retrospectively collected from emergency room (ER) charts after first assessments. Follow-up was performed in telemedicine consultation.

Setting The single-centre study was performed in a hospitalisation ward at a university hospital.

Participants Consecutive patients meeting hospitalisation criteria for COVID-19 pneumonia were eligible. Study exclusion criteria were patients who could not speak, had previous gustatory and olfactory dysfunctions or whose PCR tests for SARS-CoV-19 were negative.

Interventions Systematic assessment of gustatory and olfactory symptoms with standardised questions. Outcome(s) Prevalence of gustatory and olfactory dysfunctions in patients with COVID-19 pneumonia. Results of the 80 study subjects, $62.5 \%$ were male and the median age was 57 years. Half of the cohort $(n=40)$ presented with comorbidities. The prevalence of chemosensitive disorder was $73.8 \%(n=59)(95 \%$ Cl: 63.8 to 83.8), although self-reported symptoms were recorded in only $26.3 \%(n=21)$ of patients in the ER. Gustatory and olfactory dysfunctions were observed in $58.8 \%(n=47)$ and $55 \%(n=44)$ of cases, respectively. They were also the first symptoms in $25 \%(n=20)$ of patients. Anosmia was associated with ageusia, OR: 7, 95\% Cl: 2.3 to 21.8 , $\mathrm{p}=0.001)$. No differences in clinical outcomes were observed when patients with and without gustatory and olfactory dysfunctions were compared. Recovery rates
Strengths and limitations of this study

- Prospective study design in consecutive patients with COVID-19 pneumonia.

- Prospective recovery rates of gustatory and olfactory functions in patients with COVID-19 pneumonia.

- Lack of validated questionnaires of olfactory and gustatory functions.

- Lack of quantitative assessments of olfactory and gustatory functions.

- The selected population primarily represents hospitalised patients, excluding milder cases.

were $20 \%(n=10)$ and $85 \%(n=42)$ at days 7 and 45 , respectively.

Conclusion The prevalence of gustatory and olfactory dysfunctions in COVID-19 pneumonia was much higher than in self-report. Presence of gustatory and olfactory dysfunctions was not a predictor of clinical outcomes.

\section{INTRODUCTION}

The SARS-CoV-2 has resulted in a pandemic with more than 26 million reported cases and, as last reported in September 2020, 800000 deaths ${ }^{1}$. With no effective vaccine available, basic preventive strategies against transmission of the highly contagious virus include the early recognition of potential clinical signs. Such a strategy could minimise the possibility of early transmission, as has been described in several studies.$^{2-4}$ Similarly, as per the latest recommendations of the WHO, establishing contact tracing of suspected cases could provide key clinical findings related to SARS-CoV-2 and mitigate risk of transmission $^{5}$. Finally, prompt diagnosis and 
treatment of possibly severe viral infection could improve clinical outcomes.

Within this context, gustatory and olfactory dysfunctions are unrecognised and poorly defined condition, for which establishing strong causal relationships is challenging. Viruses, however, are the most frequent pathogens with respect to aetiology. For example, Coronavirus, Influenza and Picornavirus have been detected in nasal secretions in smell studies, with various degrees of severity and an even permanent loss of smell and taste. ${ }^{6}$ Consequently, gustatory and olfactory dysfunctions lead to a decrease in the quality of life and mood state. ${ }^{7}$

When initial reports of the SARS-CoV-2 outbreak in Wuhan area were published, gustatory and olfactory dysfunctions were not described ${ }^{8-10}$ However, a small retrospective study performed in Milan estimated a prevalence of gustatory and olfactory dysfunctions of around $34 \%$ of hospitalised patients with COVID-19 infection. ${ }^{11}$ Similarly, several other reports of isolated sudden onset anosmia have recently been described in patients with COVID-19. ${ }^{12}{ }^{13}$ Finally, a multicentre, cross-sectional study performed in France and Belgium reported a prevalence of gustatory and olfactory dysfunctions of $85 \%$ in mild cases of COVID-19. ${ }^{14}$

However, incidence of gustatory and olfactory dysfunctions has yet to be defined in severe cases of COVID-19 requiring hospital admission. Since the onset of the pandemic in Barcelona, a great number of gustatory and olfactory symptoms have been self-reported by patients admitted to our healthcare centre for confirmed COVID-19 pneumonia. The present study aims to estimate the prevalence, clinical traits and evolution of gustatory and olfactory dysfunctions in patients hospitalised for COVID-19 pneumonia.

\section{MATERIALS AND METHODS}

\section{Study design}

We performed a prospective assessment on patients consecutively admitted to a dedicated ward where attending physicians evaluated gustatory and olfactory dysfunctions. After initial evaluations, a retrospective review of emergency room (ER) charts prior to admission was also performed to determine self-report. Negative findings were determined by the absence of the studied symptoms on ER charts, whereas positive findings were determined by the presence of studied symptoms on ER charts. The study was conducted at Hospital Clínic of Barcelona, a tertiary university reference centre. The study population comprised patients with COVID-19 pneumonia admitted to a hospitalisation ward between 28 February and 24 April 2020. Data collection was performed on 30 April 2020.

The main objective of this study was to determine the proportion of gustatory and olfactory dysfunctions in hospitalised patients with confirmed COVID-19 pneumonia. Secondary endpoints were to define clinical traits, describe laboratory values, identify factors associated with gustatory and olfactory dysfunctions, explore potential differential outcomes between patients with and without gustatory and olfactory dysfunctions and examine time to recovery rates from gustatory and olfactory dysfunctions.

\section{Patients}

SARS-CoV-2 infection was confirmed by either real-time PCR viral detection in a nasopharyngeal swab or by clinical and radiological characteristics set forth by ECDC criteria, ${ }^{15}$ including: (1) acute respiratory tract infection (sudden onset of cough, fever and shortness of breath) and (2) severe acute respiratory infection (fever and at least one sign/symptom of respiratory disease (eg, cough, fever or shortness breath)) requiring hospitalisation with unilateral or bilateral interstitial infiltrate in the chest X-ray. This clinical definition was set on 15 March 2020, when the percentage of microbiologically confirmed cases of COVID-19 from all collected samples was higher than $70 \%$. Patients with prior dysfunction, unable to speak, with mental impairment or who tested negative for SARS-CoV-2 were excluded from the analysis. Additionally, to avoid memory bias, patients with direct admission to the intensive care unit (ICU) or posterior discharge to the ward were excluded. Inclusion of consecutive patients from the same ward was established to avoid selection bias.

\section{Data collection}

Directed anamnesis was performed in each patient on arrival to the ward, including baseline characteristics, demographics and classic pneumonia signs. Patients were asked about gustatory and olfactory dysfunction symptoms, such as ageusia, dysgeusia, anosmia and hyposmia on a daily basis until discharge. The following questions were asked systematically to all patients: (1) have you noticed any sudden and recent changes in smell and

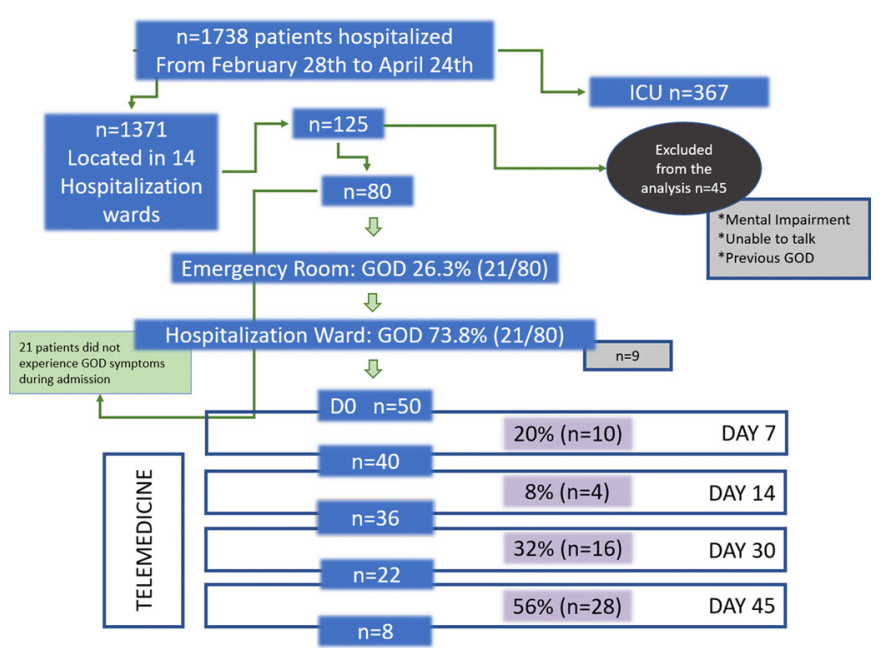

Figure 1 Study flow chart. Day 0: if the patient meets criteria included in the study. Blue boxes represent individuals on follow-up with gustatory-olfactory dysfunctions, lilac boxes represent individuals in which the recovery event develops. Grey boxes represent individuals lost to follow-up. White boxes represent timelines and mode of follow-up. 
Table 1 Baseline characteristics according to the presence of gustatory and olfactory dysfunctions in patients with COVID-19 pneumonia

\begin{tabular}{|c|c|c|c|c|}
\hline Variable & Cohort & GOD & Absence of GOD & $P$ value \\
\hline $\mathrm{N}$ & 80 & 59 & 21 & \\
\hline Median (IQR) age & $57(42-70)$ & $58(44-69)$ & $55(38-72)$ & 0.782 \\
\hline Male, $\mathrm{n}(\%)$ & $50(63)$ & $34(59)$ & $15(71)$ & 0.325 \\
\hline RT-PCR (+) nasopharyngeal swab, n (\%) & $73(91.2)$ & $55(93)$ & $19(86)$ & 0.985 \\
\hline European, n (\%) & $57(71)$ & $37(64)$ & $14(67)$ & 0.940 \\
\hline Coexisting illness, n (\%) & $40(50)$ & $27(47)$ & $12(57)$ & 0.370 \\
\hline Hypertension, $\mathrm{n}(\%)$ & $22(29)$ & $17(29)$ & $5(23)$ & 0.738 \\
\hline Diabetes, n (\%) & $8(10)$ & $6(10)$ & $2(10)$ & 0.932 \\
\hline Pulmonary disease, $\mathrm{n}(\%)$ & $8(10)$ & $7(14)$ & $1(5)$ & 0.168 \\
\hline Oncological disease, $\mathrm{n}(\%)$ & $5(6)$ & $2(3)$ & $3(14)$ & 0.76 \\
\hline Heart disease, $n(\%)$ & $5(6)$ & $3(5)$ & $2(11)$ & 0.453 \\
\hline Healthcare worker, n (\%) & $11(14)$ & $3(14)$ & $3(14)$ & 0.934 \\
\hline Median (IQR) days from onset to ER & $8(5.25-10)$ & $8(5-11)$ & $7(5.5-9)$ & 0.135 \\
\hline Fever, n (\%) & $71(89)$ & $52(88)$ & $19(91)$ & 0.771 \\
\hline Cough, n (\%) & $62(78)$ & $49(83)$ & 13(62) & 0.046 \\
\hline Shortness of breath, $n(\%)$ & $52(65)$ & $35(59)$ & $17(81)$ & 0.074 \\
\hline Asthenia, $\mathrm{n}(\%)$ & $45(56)$ & $33(56)$ & $12(57)$ & 0.934 \\
\hline Myalgia, n (\%) & $40(50)$ & $34(53)$ & $9(43)$ & 0.147 \\
\hline Diarrhoea, n (\%) & $35(44)$ & $25(42)$ & $10(48)$ & 0.677 \\
\hline Hyporexia, n (\%) & $33(38)$ & $29(49)$ & $4(19)$ & 0.016 \\
\hline Headache, n (\%) & $28(35)$ & $24(41)$ & $4(19)$ & 0.061 \\
\hline Arthralgia, n (\%) & $10(13)$ & $8(14)$ & $2(10)$ & 0.545 \\
\hline Chest pain, $\mathrm{n}(\%)$ & $9(11)$ & $6(10)$ & $3(14)$ & 0.608 \\
\hline Odynophagia n, (\%) & $7(9)$ & $6(10)$ & $1(5)$ & 0.451 \\
\hline Vomiting, n (\%) & $7(9)$ & $6(10)$ & $1(5)$ & 0.451 \\
\hline Rash, n (\%) & $5(6)$ & $5(9)$ & 0 & 0.168 \\
\hline Rhinorrhea & 2 (3) & $1(2)$ & 1 (5) & - \\
\hline
\end{tabular}

Bold formatting indicates statistical significance.

ER, emergency room; GOD, gustatory and olfactory dysfunctions.

taste?; (2) if the response was affirmative, was this the first symptom?; (3) is there a total loss of taste and smell; (4) is there a partial loss of taste and smell?; (5) have the symptoms disappeared? and (6) when did the symptoms disappear? Gustatory and olfactory dysfunctions were re-evaluated during telemedicine consultations on a weekly basis after discharge. The following questions were then asked systematically to all discharged patients: (1) was there resolution of symptoms?; (2) was there partial or total recovery? and (3) when did symptoms resolve? Additional laboratory tests (liver enzymes, creatinine, C-reactive protein, leucocyte count, CD4, CD8 and CD3 count, ferritin and D-dimer levels and procalcitonin) were performed in all patients. The last follow-up date was 24 April 2020.

For data collection, variables were extracted from electronic health records in the SAP 740 Hospital Information System (Societas Europaea, Walldorf, Germany).
The results obtained were included in a database created with MICROSOFT EXCEL for later analysis with statistical package SPSS V.18.0 (IBM corporation, Armonk, New York, USA).

\section{Statistical analysis}

The primary endpoint of the study was to determine the proportion of patients with gustatory and olfactory dysfunctions, reflected in percentages with a confidence level of $95 \%$. The Fisher's exact test was performed to analyse categorical variables between groups; significant $p$ values were shown in ORs and CIs. The significance level was set at a $p$ value $<0.05$. Inferential analysis of continuous variables, such as laboratory values, was performed using parametric tests (Student's t-test); Pearson test was performed to correlate the duration of symptoms and inflammatory markers. Missing data were reduced by the use of an operator manual at the start of the study and 
careful data collection. Missing data were managed with the listwise approach.

\section{Sample size calculation}

An estimated sample size of 80 patients would allow for an expected prevalence of $33 \%$ at a $10.5 \%$ accuracy rate and $95 \% \mathrm{CI}$, as has been shown in a prior study by Giacomelli et $a l^{11}$

\section{Ethics}

Processing, reporting and transferring of personal data for all participating subjects complied with provisions in Organic Act 15/1999 of 13 December (Spanish Royal Decree 1720/2007 of 21 December) and in current Regulation EU 2016/679 of the European Parliament and the European Council on 27 April 2016, being mandatory after 25 May 2018, on the Protection of Personal Data and guarantee of digital rights. The patients either signed written consent or when not possible, due to the pandemic and isolation measures in place, provided oral consent. In such cases, oral consent was recorded in Electronic Clinical Documentation.

\section{Patient and public involvement}

The patients were not involved in the study described.

\section{RESULTS}

\section{Baseline characteristics}

Of the 1738 patients with COVID-19 admitted to the hospital between 28 February and 24 April, 1371 were placed in several hospitalisation wards and 367 in the ICU. Analysis of gustatory and olfactory dysfunctions was prospectively performed in 80 patients (figure 1 flow chart). The median (IQR) age was 57 (43-70) years and $62.5 \%$ were males. Eleven (14\%) patients were healthcare workers. Continent origin of patients comprised $71 \%(\mathrm{n}=57)$ European primarily, and 25\% $(\mathrm{n}=20)$ Latin American. Comorbidities were present in $50 \%(\mathrm{n}=40)$ of patients. The median (IQR) time from symptom onset to hospital admission was $8^{5-10}$ days, and the median (IQR) length of stay (LOS) was 8 (5-12.5) days (table 1$)$.

\section{Gustatory and olfactory dysfunction}

The main clinical symptoms reported by patients are depicted in table 1 . The prevalence of gustatory and olfactory dysfunctions was found to be $73.8 \% \quad(n=59)$ (95\% CI: $63.8 \%$ to $83.8 \%$ ) in this sample. Separately, gustatory and olfactory dysfunctions was present in $58.8 \%(n=47)$ and $55 \%(n=44)$ of cases, respectively. Anosmia was the most frequent symptom present in $46.3 \%(\mathrm{n}=37)$ of cases, followed by dysgeusia in $41.3 \%(\mathrm{n}=33)$, ageusia in $28.3 \%(\mathrm{n}=23)$ and hyposmia in $15 \%(n=12)$. In $21 \%(n=7)$ of cases of dysgeusia, patients reported a salty taste. There was an association between ageusia and anosmia, OR: 7 (95\% CI: 2.26 to $21.8, p=0.001)$. Gustatory and olfactory

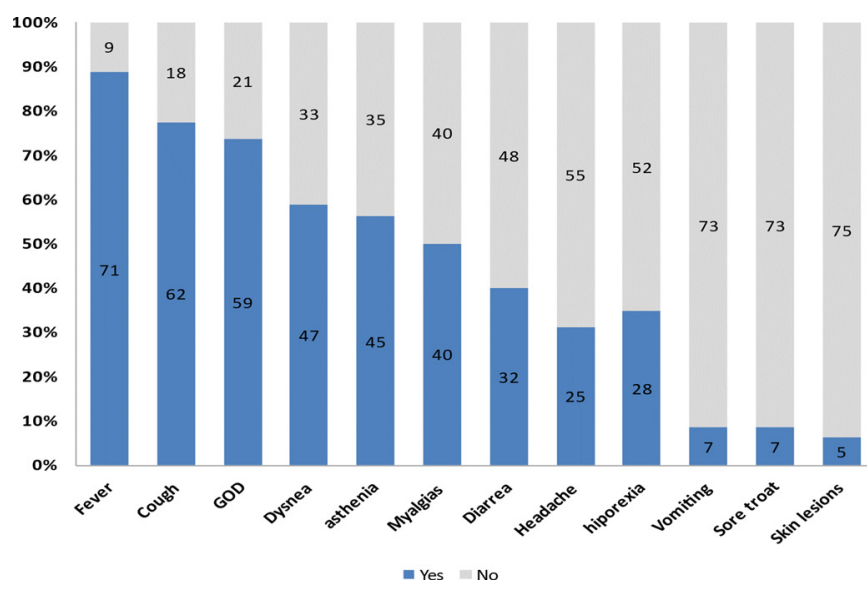

Figure 2 General symptoms in patients with COVID-19 pneumonia in absolute numbers $(n=80), G O D$, gustatory and olfactory dysfunctions.

dysfunctions was the first symptom to appear in $25 \%$ $(n=20)$ of cases. Only one case presented with gustatory and olfactory dysfunctions 7 days after admission; the remaining patients presented with gustatory and olfactory symptoms prior to hospital admission. In the ER, only $26.3 \%(n=21)$ of patients reported olfactory-gustatory dysfunctions. ER physicians were more likely to report positive than negative clinical findings $(90.5 \%$ vs $9.5 \%$, respectively), OR: 4.6 (0.97 to 21.95: $95 \% \mathrm{CI}, \mathrm{p}=0.039)$. Among classic pneumonia symptoms, fever was present in $89 \%(n=71)$ of patients. It was also the most frequent consulted symptom in $53.8 \%(\mathrm{n}=43)$ of patients who visited the ER. The second most common symptom was coughing, being present in $77.5 \% \quad(\mathrm{n}=62)$ of cases, followed by dyspnoea in $58.8 \%(47 \%)$, asthenia in $56.3 \%(\mathrm{n}=45)$, myalgia in $50 \%(\mathrm{n}=40)$, hyporexia in $38 \%(\mathrm{n}=33)$, vomiting in $8.8 \%(\mathrm{n}=7)$ and skin lesions in $6.3 \%(\mathrm{n}=5)$ (figure 2). Vomiting and skin lesions were uncommon. Furthermore, hyporexia was associated with gustative dysfunction, OR: 4.1 (1.23 to 13.67: $95 \% \mathrm{CI}, \mathrm{p}=0.016)$. An association between cough and the presence of gustatory and olfactory dysfunctions was also observed, OR: 1.2 (0.12 to 12.86 : $95 \% \mathrm{CI}$, $\mathrm{p}=0.046)$ (table 1).

As per laboratory abnormalities, C-reactive protein values were elevated on admission in $91.8 \%$ of cases; lymphopoenia was present in $98.8 \%$ of cases. Other common findings included elevated levels of lactate dehydrogenase $(76.2 \%$ of cases), D-dimer $(66.2 \%)$ and serum ferritin $(63.2 \%)$ (table 2 ).

Differences between aspartate aminotransferase and alanine transferase values were observed between patients with gustatory-olfactory dysfunctions and those without symptoms ( 33 vs $61 \mathrm{UI} / \mathrm{l}) \mathrm{p}=0.019$ and (28 vs 49) $\mathrm{p}=0.003$, respectively). No differences in other laboratory findings were found between the studied groups (table 2). 
Table 2 Laboratory values according to the presence of gustatory and olfactory dysfunctions in patients with COVID-19 pneumonia

\begin{tabular}{|c|c|c|c|c|}
\hline Variable & Cohort & GOD & Absence of GOD & $P$ value \\
\hline $\mathrm{N}$ & 80 & 59 & 21 & \\
\hline CRP mg/dL $(<1.0)$ & $9(3-14)$ & $8(3-16)$ & $10(5-13)$ & 0.838 \\
\hline LDH mg/dL (<234) & $310(268-386)$ & $309(250-367)$ & $342(260-453)$ & 0.109 \\
\hline Ferritin mg/dL (<200) & $716(256-1322)$ & $592(241-1255)$ & $1066(300-1552)$ & $0-093$ \\
\hline $\begin{array}{l}\text { AST UI/L } \\
(5.0-40.0)\end{array}$ & $39(25-61)$ & $33(22-50)$ & $61(39-94)$ & 0.003 \\
\hline ALT UI/L (5.0-40.0) & $29(20-68)$ & $28(19-55)$ & $49(27-102)$ & 0.019 \\
\hline BT mg/dL (0.20-1.20) & $0.64(0.8-0.4)$ & $0.6(0.4-0.75)$ & $0.8(0.55-1.05)$ & 0.560 \\
\hline LYM cells $/ \mathrm{mm}^{3}(4.00-11.00)$ & $0.9(0.6-1.3)$ & $0.9(0.6-1.3)$ & $0.8(0.6-1.5)$ & 0.820 \\
\hline D-dimer ng/mL $(<500)$ & $800(400-1200)$ & $700(400-1100)$ & $1000(600-1800)$ & 0.251 \\
\hline Platelets cells $/ \mathrm{mm}^{3}$ (130-400) & $212(168-266)$ & $213(170-265)$ & $209(150-281)$ & 0.735 \\
\hline Creatinine mg/dL (0.30-1.30) & $0.6(0.7-09)$ & $0.78(0.63-0.91)$ & $0.86(68-0.98)$ & 0.630 \\
\hline Calcium meq/L (8.5-9.5) & $8.2(8-8.5)$ & $8.2(8-8.6)$ & $8.1(7.8-8.3)$ & 0.735 \\
\hline 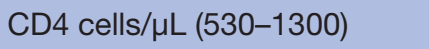 & $356(248-579)$ & $358.5(258.7-630)$ & $328(225-464)$ & 0.414 \\
\hline 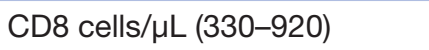 & $225(147-353)$ & $241(148-256)$ & $186(140.7-396)$ & 0.934 \\
\hline CD3 cells/ $\mu \mathrm{L}$ (1000-2200) & $630(404-1014)$ & $676(459.5-1035)$ & 496 (386-792) & 0.310 \\
\hline
\end{tabular}

ALT, Alanine transferase; AST, Aspartate aminotransferase ; Bold formatting, indicates statistical significance; BT, total bilirubin; CD4, CD4+count; CD8, CD8+count; CRP, C-reactive protein; GOD, gustatory and olfactory dysfunctions; LDH, lactate dehydrogenase; LYM, lymphocyte.

\section{Treatment prescribed}

All patients received hydroxychloroquine and azithromycin. In approximately $91.3 \%(\mathrm{n}=73)$ of patients, lopinavir boosted with ritonavir was administered; $38.8 \% \quad(\mathrm{n}=31)$, interleukin blockers and $30.4 \%$ $(n=24)$, corticosteroids. In $18.8 \%(n=15)$ of patients, teicoplanin was administered, whereas in $7.6 \% \quad(n=6)$ of patients, interferon was administered. Oxygen supplementation and intubation were required in $69.8 \%(\mathrm{n}=55)$ and $10 \%(5 \%)$ of patients, respectively. No differences were observed between study groups (table 3).

\section{Clinical outcomes}

A total of $90 \%(\mathrm{n}=73)$ of patients were discharged on completion of the study period and $13.8 \% \quad(n=11)$ required ICU admission during hospitalisation, of whom none died. After discharge, $87 \% \quad(n=67)$ of patients were followed up via a telemedicine consultation for any possible changes in status. No readmission episodes were recorded.

In terms of ICU admission, $\mathrm{LOS}, \mathrm{SpO}_{2} / \mathrm{FiO}_{2}$ ratio, oxygen supplementation, respiratory distress or organising pneumonia. No association was observed in outcomes between patients with gustatory and olfactory dysfunctions and patients without gustatory and olfactory dysfunctions. Pulmonary embolism was associated with the absence of gustatory and olfactory dysfunctions (21\% vs 0) p=0.0001 (table 3 ).

\section{Gustatory and olfactory outcomes}

Of the 59 patients with gustatory and olfactory dysfunctions, 50 were reassessed for follow-up. On day 7 after discharge, $20 \% \quad(n=10)$ of these patients had recovered gustatory and olfactory functions. Recovery rates at day 14,30 and 45 were $28 \%(\mathrm{n}=14), 56 \%(\mathrm{n}=28)$ and $84 \%$ $(n=42)$, respectively. The median time of olfactory-gustatory recovery was 14 (7-30) days (figure 3).

Time of olfactory-gustatory recovery was positively correlated with age $(\mathrm{r}: 0.48 \mathrm{p}=0.03)$ and ferritin levels $(\mathrm{r}$ : $0.353 \mathrm{p}=0.037$ ). No correlations with severity of respiratory function or any other abnormal laboratory values were found.

\section{DISCUSSION}

This is a prospective study evaluating the prevalence of gustatory and olfactory dysfunctions in hospitalised patients. We found a prevalence of $74 \%$ in all patients admitted to a hospital ward with COVID-19 pneumonia who could be questioned. In a previous study in hospitalised patients, overall prevalence was reported at $33 \% .^{11}$ The main reasons for this discrepancy could be attributed to the retrospective design of that study and an unidentified prevalence of gustatory and olfactory dysfunctions of $53 \%$ in this prospective cohort due to reassessment of ER electronic health records in hospitalisation wards. In fact, brief communication reports in Italy have already 
Table 3 Clinical outcomes according to the presence of gustatory and olfactory dysfunctions in patients with COVID-19 pneumonia

\begin{tabular}{|c|c|c|c|c|}
\hline Variable & Cohort & GOD & Absence of GOD & $P$ value \\
\hline $\mathrm{N}$ & 80 & 59 & 21 & \\
\hline LOS (IQR) & $8(4-11.75)$ & $8(4.2-11)$ & $7(4-14)$ & 0.836 \\
\hline $\mathrm{SpO}_{2} / \mathrm{fiO}_{2}$ ratio (IQR) & $448(245-247)$ & $451(257-471)$ & $391(197-471)$ & 0.346 \\
\hline Lopinavir/ritonavir, n (\%) & $73(94)$ & $52(91)$ & $21(100)$ & 0.161 \\
\hline Interferon, $\mathrm{n}(\%)$ & $6(8)$ & $4(7)$ & $2(10)$ & 0.638 \\
\hline Corticosteroid, n (\%) & $24(30)$ & $15(3)$ & $9(43)$ & 0.147 \\
\hline Interleukin blocker, n (\%) & $33(41)$ & $22(37)$ & $11(52)$ & 0.228 \\
\hline ICU admission, n (\%) & $11(14)$ & $7(12)$ & $4(19)$ & 0.412 \\
\hline Complication n, (\%) & $32(41)$ & $20(35)$ & $12(57)$ & 0.07 \\
\hline Oxygen requirement, n (\%) & $55(69)$ & $40(68)$ & $15(71)$ & 0.758 \\
\hline Orotraqueal intubation, $\mathrm{n}(\%)$ & 8 & $5(8.5)$ & $3(14)$ & 0.867 \\
\hline Respiratory distress, n (\%) & 31 & $20(34)$ & $11(52)$ & 0.135 \\
\hline Pulmonary embolism, n (\%) & $5(6)$ & 0 & $5(23)$ & 0.0001 \\
\hline Organising pneumonia, n (\%) & $10(13)$ & $5(9)$ & $5(24)$ & 0.068 \\
\hline
\end{tabular}

$\mathrm{FiO}_{2}$, fraction of inspired oxygen; GOD, gustatory and olfactory dysfunctions; ICU, intensive care unit; LOS, length of stay; $\mathrm{SpO}_{2}$, peripheral capillary oxygen saturation.

acknowledged a self-reported prevalence of gustatory and olfactory dysfunctions of $19 \%$ in retrospective charts. ${ }^{16}$

In our cohort, gustatory and olfactory dysfunctions syndrome was almost as prevalent as fever and cough, two classic symptoms of viral pneumonia. Within the context of flu-like symptoms and gustatory-olfactory dysfunctions syndrome, the possibility of COVID-19 infection is worth considering. In a recent case-control study, gustatory and olfactory dysfunctions were 10 times more common in patients with positive PCR testing results than in those who tested negative for the COVID-19 infection. ${ }^{17}$

Our study reported anosmia in $55 \%$ of cases, whereas investigators, Lechein et al, reported anosmia in $86 \%$ of cases within the setting of a symptom-oriented, otorhinolaryngology-specialised consultation. Patients in that study were younger, without comorbidities and primarily healthcare providers, contrasting our older

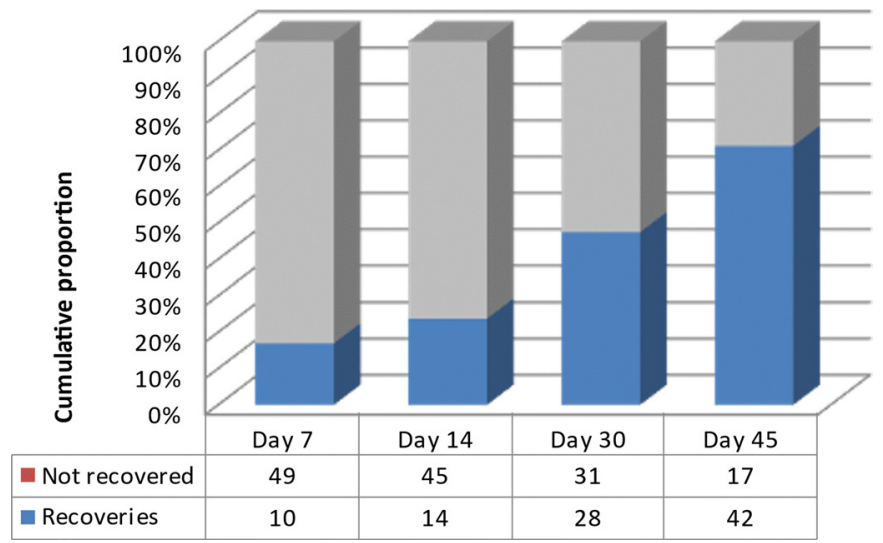

Figure 3 Recovery rates of gustatory and olfactory dysfunctions trough time at follow-up postdischarge $(n=50)$. cohort of hospitalised patients who presented with more comorbidities. Similarly, with results being obtained by quantitative testing of sense of smell, an Iranian casecontrol study reported olfactory changes in nearly $98 \%$ of patients with COVID-19 and a median age of 46 years. This study suggests that quantitative testing of hyposmia might serve as a rapid, alternative diagnostic approach for SARS-CoV-2 screening. ${ }^{18}$ However, the role of this test in older patients remains unclear given age-related changes that occur in olfactory function. ${ }^{19}$

Similar clinical signs and symptoms were observed between patients with and without gustatory and olfactory dysfunctions. Coughing was observed more frequently in patients with gustatory and olfactory dysfunctions, whereas a more severe respiratory syndrome was not. Gustatory dysfunction was associated with hyporexia, which could affect a patient's quality of life and daily activities especially in cases of persistence. ${ }^{20} 21$

Additionally, no major alterations in laboratory values were observed. A mild-to-moderate elevation of liver enzymes were common in the absence of gustatory and olfactory dysfunction symptoms; no case of acute liver failure was reported. Although an explanation for these findings is difficult to provide, this observation may be due to inflammatory changes in the liver or in relation to a different tissue expression of receptors and entry molecules. ACE2 and TMPRSS2 are expressed in cells from multiple tissues, including olfactory cells and liver; the degree to which tropism might affect different tissues has not been well established. ${ }^{22}$

Clinical outcomes were similar in both groups, however pulmonary embolism occurs in $20 \%$ of patients without gustatory and olfactory dysfunction symptoms. 
Confounding factors associated with pulmonary embolism such as thromboprophylaxis could not be excluded, ${ }^{23}$ and there is some debate as to whether or not the presence of chemosensory disorder is related to a better prognosis. In some studies, the absence of anosmia was associated with a mild-to-moderate COVID-19 infection and outpatient care. ${ }^{24}$ Other studies have concluded that there is no prognostic value; however, the persistence of olfactory dysfunction on day 20 is associated with a more severe disease course. ${ }^{25}$ Disparity of these findings might be explained by bias, for example, severe and critically ill patients who are vulnerable to self-neglect and by consequence, do not recognise chemosensory loss and older patients failing to recognise an acute chemosensory loss due to a prior, unidentified olfactory impairment related to senescence. Investigators, Lechein $e t$ al, reported recovery rates of olfactory function at $72 \%$ on day 8 . Since our study represented olfactory and gustatory outcomes together, performing comparisons might be difficult; nonetheless, we found recovery rates of $20 \%$ on day 7 and $84 \%$ on day 45 . Finally, another possibility for this discrepancy is the older age and more severe clinical manifestations of COVID-19 infection in our cohort.

Although no correlation between etiopathogenic factors and the length of gustatory and olfactory functions' recovery has been made thus far, the inflammatory response might play a role. Our data support that higher levels of ferritin and older age are associated with longer recovery rates. Ferritin levels have been reported to be markedly higher in severe cases than in moderate cases of COVID-19 with longer recovery rates; older age was associated with higher disease severity. ${ }^{26-28}$

\section{Strengths and weaknesses of the study}

The main limitation of this study was the lack of validated olfactory and gustatory questionnaires based on smell and taste components, which could have provided an even more standardised approach. Another limitation was the impossibility to perform olfactory-gustatory testing using quantitative measurements and to include accepted retronasal olfaction test methods. In spite of this, a standardised, non-validated questionnaire was implemented and used systematically in all patients. Consecutive patients were included in this study to minimise selection bias, although selection could be a cause of bias in our data.

The study population selected is limited to a single centre, representing hospitalised patients mostly and excluding milder cases without pulmonary involvement and the most critical cases with direct ICU admission. Moreover, not all of the patients had microbiologically confirmed cases of infection or underwent complete follow-up after hospital discharge.

\section{CONCLUSIONS AND POLICY IMPLICATIONS}

There is high prevalence of gustatory and olfactory dysfunctions in hospitalised patients with COVID-19 pneumonia. Self-reporting rates are low in our population. Clinical outcomes were not related with presence or the absence of gustatory and olfactory dysfunctions in hospitalised patients.

Acknowledgements HIV Unit: Jose-Luis Blanco, Maria Martinez-Rebollar, Esteban Martinez, Josep Mallolas, Pilar Callau. All the personnel of Medical Intensive Care Unit: Josep Maria Nicolás, Pedro Castro, Sara Fernández and Adrian Téllez. Department of International Health: Alex Almuedo-Riera, Jose Muñoz, Daniel Camprubi Ferrer, Maria Teresa de Alba, Marc Fernandez, Elisabet Ferrer, Berta Grau, Helena Marti, Magdalena Muelas, Maria Jesus Pinazo, Natalia Rodríguez, Montserrat Roldan, Júlia Garcia-Pindado, Carme Subira, Isabel Vera, Nana Williams. We would also like to thank Anthony Armenta for providing medical editing assistance in English.

Contributors Al, CC, MC, PA, BT, AGC, VR, DA, NG-P, MH-M, LA, JR, FM, LDLM, LL, PP-A, IM, ML, JA, AR, LM, MB, EM-G, AM and MS performed clinical assessments. $\mathrm{Al}$ and $\mathrm{FG}$ designed the study, contributed to data analysis and wrote the first draft of the manuscript. Al, CC, MC, LL and MT were responsible for data entry. Al, CG-V, JAM, IA, AS, and FG critically reviewed the manuscript and agreed on its final version.

Funding The authors have not declared a specific grant for this research from any funding agency in the public, commercial or not-for-profit sectors.

Competing interests None declared.

Patient and public involvement Patients and/or the public were not involved in the design, or conduct, or reporting or dissemination plans of this research.

Patient consent for publication Not required.

Ethics approval The hospital's research ethics committee and competent Spanish authorities approved the protocol describing the project proposed by the researcher (approval number HCB/2020/0458).

Provenance and peer review Not commissioned; externally peer reviewed.

Data availability statement Data are available upon reasonable request. All data relevant to the study are included in the article or uploaded as supplementary information. Extra data will be available by emailing ajinciar@clinic.cat Data are available upon reasonable request.

Open access This is an open access article distributed in accordance with the Creative Commons Attribution Non Commercial (CC BY-NC 4.0) license, which permits others to distribute, remix, adapt, build upon this work non-commercially, and license their derivative works on different terms, provided the original work is properly cited, appropriate credit is given, any changes made indicated, and the use is non-commercial. See: http://creativecommons.org/licenses/by-nc/4.0/.

ORCID iDs

Alexy Inciarte http://orcid.org/0000-0003-1896-3457

Estela Moreno-García http://orcid.org/0000-0002-3205-2185

\section{REFERENCES}

1 COVID-19 coronavirus pandemic. Available: https://www. worldometers.info/coronavirus/ [Accessed 05 Sep 2020].

2 Nishiura H, Kobayashi T, Suzuki A. Estimation of the asymptomatic ratio of novel coronavirus infections (COVID-19). Int $J$ Infect Dis 2020;S1201-9712:30139-9.

3 Bai Y, Yao L, Wei T, et al. Presumed asymptomatic carrier transmission of COVID-19. JAMA 2020;323:1406-7.

4 Zhang J, Tian S, Lou J, et al. Familial cluster of COVID-19 infection from an asymptomatic. Crit Care 2020;24:119.

5 WHO. COVID-19: operational guidance for maintaining essential health services during an outbreak. Available: https://www.who.int/ publications-detail/covid-19-operational-guidance-for- maintainingessential-health-services-during-an-outbreak [Accessed $30 \mathrm{Apr}$ 2020].

6 Suzuki M, Saito K, Min W-P, et al. Identification of viruses in patients with postviral olfactory dysfunction. Laryngoscope 2007;117:272-7.

7 Smeets MAM, Veldhuizen MG, Galle S, et al. Sense of smell disorder and health-related quality of life. Rehabil Psychol 2009;54:404-12.

8 Wang Z, Yang B, Li Q, et al. Clinical features of 69 cases with coronavirus disease 2019 in Wuhan, China. Clin Infect Dis 2020;71:769-77. 
9 Huang C, Wang Y, Li X, et al. Clinical features of patients infected with 2019 novel coronavirus in Wuhan, China. Lancet 2020;395:497-506.

10 CDC Weekly $C$. The epidemiological characteristics of an outbreak of 2019 novel coronavirus diseases (COVID-19) China, 2020. China CDC Weekly 2020;2:113-22.

11 Giacomelli A, Pezzati L, Conti F, et al. Self-Reported olfactory and taste disorders in patients with severe acute respiratory coronavirus 2 infection: a cross-sectional study. Clin Infect Dis 2020;71:889-90.

12 Ollarves-Carrero MF, Rodriguez-Morales AG, Bonilla-Aldana DK, et al. Anosmia in a healthcare worker with COVID-19 in Madrid, Spain. Travel Med Infect Dis 2020;35:101666.

13 Gane SB, Kelly C, Hopkins C. Isolated sudden onset anosmia in COVID-19 infection. A novel syndrome? Rhinology 2020;58:299-301.

14 Lechien JR, Chiesa-Estomba CM, De Siati DR, et al. Olfactory and gustatory dysfunctions as a clinical presentation of mild-to-moderate forms of the coronavirus disease (COVID-19): a multicenter European study. Eur Arch Otorhinolaryngol 2020;277:2251-61.

15 Case definition and European surveillance for COVID-19, 2020. Available: https://www.ecdc.europa.eu/en/case-definition-andeuropean-surveillance-human-infection- novel-coronavirus-2019ncov [Accessed 30 Apr 2020].

16 Vaira LA, Salzano G, Deiana G, et al. Anosmia and Ageusia: common findings in COVID-19 patients. Laryngoscope 2020;130:1787.

17 Yan CH, Faraji F, Prajapati DP, et al. Association of chemosensory dysfunction and COVID-19 in patients presenting with influenza-like symptoms. Int Forum Allergy Rhinol 2020;10:806-13.

18 Moein ST, Hashemian SM, Mansourafshar B, et al. Smell dysfunction: a biomarker for COVID-19. Int Forum Allergy Rhinol 2020;10:944-50.

19 Doty R, Shaman P, Applebaum S, et al. Smell identification ability: changes with age. Science 1984;226:1441-3.
20 Miwa T, Furukawa M, Tsukatani T, et al. Impact of olfactory impairment on quality of life and disability. Arch Otolaryngol Head Neck Surg 2001;127:497-503.

21 Rusthen S, Young A, Herlofson BB, et al. Oral disorders, saliva secretion, and oral health-related quality of life in patients with primary Sjögren's syndrome. Eur J Oral Sci 2017;125:265-71.

22 Sungnak W, Huang N, Bécavin C, et al. SARS-CoV-2 entry factors are highly expressed in nasal epithelial cells together with innate immune genes. Nat Med 2020;26:681-7.

23 Tang $\mathrm{N}$, Bai $\mathrm{H}$, Chen $\mathrm{X}$, et al. Anticoagulant treatment is associated with decreased mortality in severe coronavirus disease 2019 patients with coagulopathy. J Thromb Haemost 2020;18:1094-9.

24 Yan CH, Faraji F, Prajapati DP, et al. Self-reported olfactory loss associates with outpatient clinical course in COVID-19. Int Forum Allergy Rhinol 2020;10:821-31.

25 Vaira LA, Hopkins C, Petrocelli M, et al. Do olfactory and gustatory psychophysical scores have prognostic value in COVID-19 patients? A prospective study of 106 patients. J Otolaryngol Head Neck Surg 2020;49:56.

26 Ruan Q, Yang K, Wang W, et al. Clinical predictors of mortality due to COVID-19 based on an analysis of data of 150 patients from Wuhan, China. Intensive Care Med 2020;46:846-8.

27 Yang $X, Y u$ Y, Xu J, et al. Clinical course and outcomes of critically ill patients with SARS-CoV-2 pneumonia in Wuhan, China: a singlecentered, retrospective, observational study. Lancet Respir Med 2020;8:475-81.

28 Zhou F, Yu T, Du R, et al. Clinical course and risk factors for mortality of adult inpatients with COVID-19 in Wuhan, China: a retrospective cohort study. Lancet 2020;395:1054-62. 